\title{
INFLUENCE DU CHAUFFAGE SUR LE FACTEUR ANTIBIOTIQUE PRÉSENT DANS LES MIELS
}

PAR

\section{GONNET et P. LAVIE}

Station expérimentale d'Apiculture, Montfavet.

Depuis très longtemps les propriétés antiseptiques du miel sont commues et, an cours de l'histoire, l'homme s'est fréquemment servi de ce produit naturel soit pour conserver des denrées alimentaires durant 1'hiver ou au cours des grands voyages sur mer, soit pour soigner diverses affections. Dans des temps plus anciens les hommes se servaient du miel potrr embaumer les cadavres. Le miel, qui devrait être pollué de germes innombrables, ne fermente pas habituellement cependant dans la ruche où la température (voisine de $34^{\circ}$ en certains points), l'hygrométrie et l'obscurité sont autant de facteurs favorables au développement bactérien et fongique. Des essais ultérieurs, au laboratoire, ont confirmé également ces constatations. Plus récemment des observations ont montré que le nombre de germes trouvés dans le miel est toujours peu élevé. On ne rencontre en effet, en dehors des champignons et des levures, aucune bactérie sous sa forme végétative. Dès I906, WHITE constatait la stérilité du miel provenant d'une ruche saine. Ises spores de bactéries présentes dans le miel (GABBER'T, I936) sont très souvent des germes banaux du type subtilis, plus rarement des diplocoques non pathogènes, mais l'on n'y trouve jamais d'entérobactériacées.

Les propriétés antiputrides du miel connues empiriquement sont depuis une trentaine d'années vérifiées scientifiquement, principalement grâce aux travaux fort nombreux de l'Ecole allemande. C'est alors que l'on a rejeté 1'hypothèse qui attribuait la stabilité du miel à la seule infuence des sucres ou à l'acidité et que l'on a établi la présence d'un facteur antibiotique dans le miel, nommé par Doln " l'inhibine ». Il semblait au premier examen que le sujet fut en partie épuisé, mais ayant repris la question depuis quelques années nous nous sommes aperçus qu'il n'en était rien et que l'étude du facteur antibiotique du miel est 
complexe et se présente sous de multiples aspects. On peut envisager plusieurs directions de recherches importantes : origine du facteur antibiotique, sa constance dans les différents miels, modes d'extraction et de fractionnement, influence des facteurs physiques externes, conservation lors du vieillissement du miel, influence $d \mathfrak{p H}$, action antagoniste d'un facteur de croissance. Nous avons entrepris des recherches sur l'ensemble de la question (I). L,es résultats obtenus dès à présent, à notre surprise ne cadrent pas avec ce qui semblait bien établi depuis de nombreuses années. Cette note ne traitera que d'un sujet unique, très important, à savoir : la thermostabilité du facteur antibiotique dans les miels.

\section{A. - TRAVAUX aNterieURS.}

En ce qui concerne le facteur antibiotique des miels, les notions de thermostabilité et de photolabilité ont été mises en évidence par DoLD et reprises ensuite par de nombreux auteurs : DOLD ; DU et DzIAo ; Dor,D et Knapp ; Dold et Witzenhausen ; Schuler. et Vogel ; Franco : Franco et Sartori ; Duisberg et Gebelein ; Vergí ; Póthmann ; SCHADE, Marsh et ECKERT. Seuls Dold, DU et Dziao donnent des tableaux précis et semblent avoir étudié d'une manière systématique la température et le temps de chauffage. Ises tableaux I et II indiquent quelques résultats obtenus par ces auteurs.

Tableau I

D'après Dold, Du el Dziao

\begin{tabular}{|c|c|c|c|c|c|c|c|c|c|}
\hline \multirow{2}{*}{$\begin{array}{c}\text { Souches } \\
\text { bactériennes }\end{array}$} & \multicolumn{8}{|c|}{$\begin{array}{l}\text { Développement en } 24 \text { heures sur un milieu } \\
\text { avec } 17 \% \text { de miel naturel-agar }\end{array}$} & \multirow{2}{*}{$\begin{array}{l}\text { Témoin } \\
3 \% \text { arar } \\
\text { sans nitel }\end{array}$} \\
\hline & $\begin{array}{l}\text { Non } \\
\text { chauffé }\end{array}$ & $\begin{array}{l}\mathrm{I} / 2 \mathrm{~h} \\
56^{\circ} \mathrm{C}\end{array}$ & ${ }_{5^{\circ}} \mathrm{h} . \mathrm{C}$ & $\begin{array}{c}10^{\prime} \\
70^{\circ} \mathrm{C}\end{array}$ & $80^{\circ} \mathrm{C}$ & $\begin{array}{l}10^{\prime} \\
80^{\circ} \mathrm{C}\end{array}$ & $\stackrel{2^{\prime}}{\operatorname{IOO}^{0} \mathrm{C}}$ & $5^{\prime}$ & \\
\hline 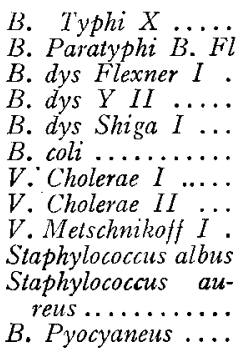 & $\begin{array}{l}\overline{+} \\
\overline{+} \\
\overline{+} \\
+ \\
\overline{-} \\
- \\
- \\
-\end{array}$ & $\begin{array}{l}+++ \\
+++ \\
+++ \\
+++ \\
+++ \\
+++ \\
+- \\
+- \\
+- \\
+++ \\
t++ \\
+++\end{array}$ & $\begin{array}{l}+++ \\
+++ \\
+++ \\
+++ \\
+++ \\
+++ \\
+++ \\
+++ \\
+++ \\
+++ \\
+++ \\
+++\end{array}$ & $\begin{array}{l}++ \\
++ \\
++ \\
++ \\
++ \\
++ \\
++ \\
++ \\
++ \\
++ \\
+++ \\
++\end{array}$ & $\begin{array}{l}++ \\
++ \\
+ \\
++ \\
++ \\
+++ \\
++ \\
++ \\
++ \\
++ \\
+++ \\
+++\end{array}$ & $\begin{array}{l}+++ \\
+++ \\
+++ \\
+-+ \\
+++ \\
+++ \\
+++ \\
+++ \\
+++ \\
+++ \\
+++ \\
+++\end{array}$ & $\begin{array}{l}++ \\
++ \\
++ \\
++ \\
++ \\
+++ \\
+ \\
++ \\
++ \\
++ \\
+ \\
+++\end{array}$ & $\begin{array}{l}+++ \\
+++ \\
+++ \\
+++ \\
+++ \\
+++ \\
+++ \\
+++ \\
+++ \\
+++ \\
+++ \\
+++\end{array}$ & $\begin{array}{l}++++ \\
++++ \\
+++t \\
+++t \\
++++ \\
++++ \\
++++ \\
++++ \\
++++ \\
++++ \\
+++t \\
++++\end{array}$ \\
\hline $\begin{aligned}- & : \text { Auc } \\
+- & : \text { Tra } \\
+ & : \text { Dév } \\
++ & : \text { Dév } \\
+++ & : \text { Dév } \\
++++ & : \text { Dév }\end{aligned}$ & $\begin{array}{l}\text {.n dével } \\
\text { eloppeme de } \\
\text { eloppeme } \\
\text { eloppeme } \\
\text { eloppeme }\end{array}$ & $\begin{array}{l}\text { olppemen } \\
\text { evelopper } \\
\text { ent faibl } \\
\text { ent moye } \\
\text { ent fort } \\
\text { ent très }\end{array}$ & $\begin{array}{l}\text { macr } \\
\text { nent } \\
\text { fort. }\end{array}$ & jique & & & & & \\
\hline
\end{tabular}

(1) Plusieurs notes sont en cours de rédaction. 
TABLEAU II

D'après DoLD, DU et DzIAO

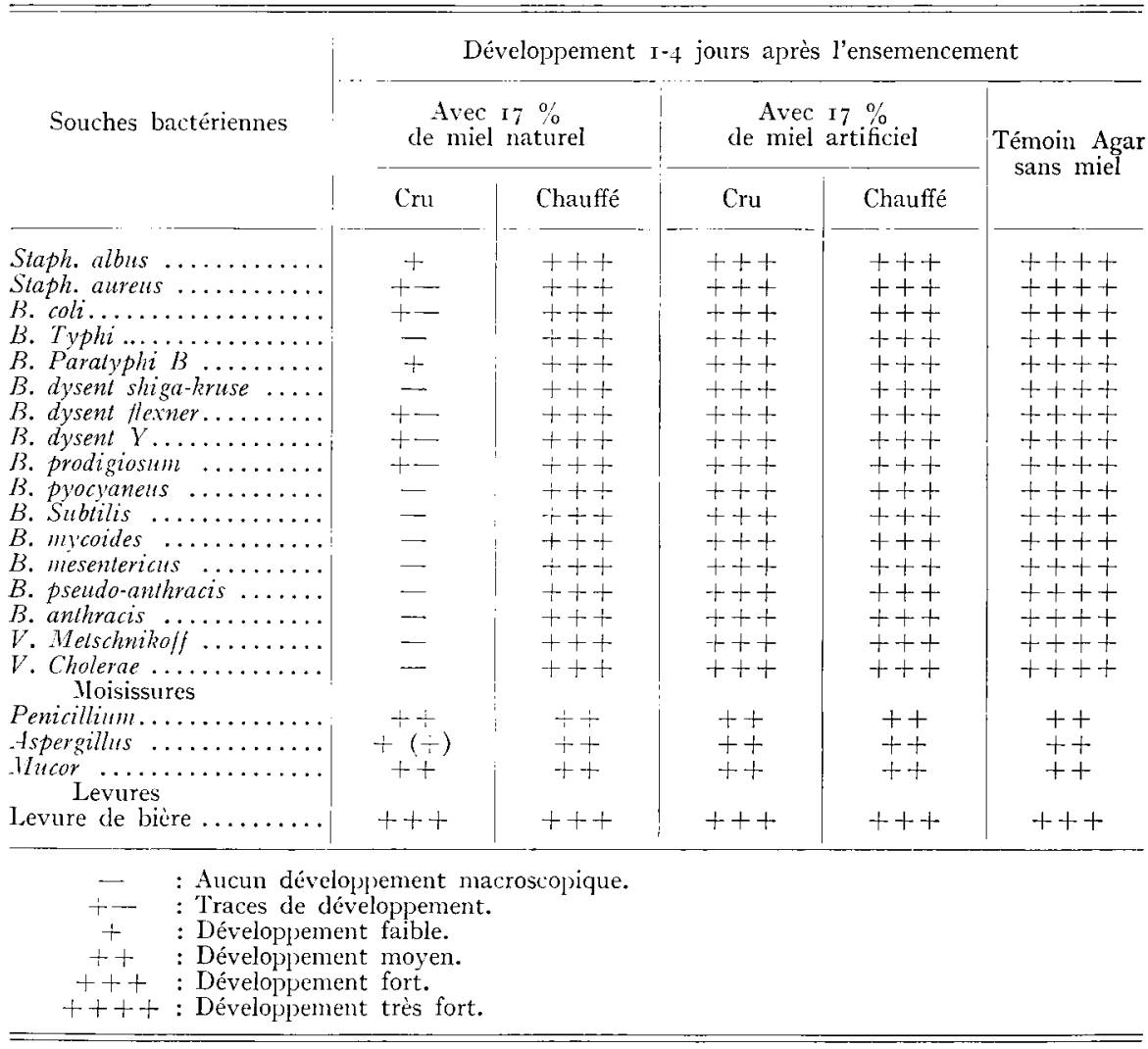

D'après DolD, Du et DzLao (I938) voici la sensibilité de l'inhibine à l'élévation de température.

Un chauffage de 30 minutes à $5^{\circ} \mathrm{C}$. suffit à détruire l'inhibine. De même,

Un chauffage de Io minutes à $70^{\circ} \mathrm{C}$. suffit à détruire d'inhibine,

Un chauffage de 5 minutes à $80^{\circ} \mathrm{C}$. suffit à détruire l'inhibine,

Un chauffage de 2 minutes à $100^{\circ} \mathrm{C}$. conduit à une destruction au moins partielle de l'inhibine.

Signalons encore que les auteurs ont mis en évidence l'inactivation de l'inhibine après exposition pendant I heure à la lumière solaire directe. D'après eux également, à 1'obscurité et à la température ordinaire du laboratoire, la conservation de $1^{\prime}$ inhibine est très bonne; mais déjà à $37^{\circ}$ elle diminue de façon perceptible après 7 à 35 jours selon les souches éprouvées. Lorsque le miel est dilué à 5o p. roo dans du sérum physiologique, l'inhibine disparaît rapidement, en 24 à $\mathrm{I} 44$ heures à l'obscurité et à la 
la température ordinaire. Les épreuves sur les bactéries ont été réalisées dans la plupart des cas de la manière suivante : on mélangeait directement le miel à essayer à un milieu gélosé et on ensemençait sur boîtes de pétri. Un autre test a également servi pour le miel : le produit était exposé aux conditions naturelles de décomposition à l'air libre. Les auteurs pouvaient alors montrer qu'un miel chauffé se défendait moins bien contre la décomposition qu'un miel frais.

Cependant, il convient de faire très attention an fait que les autenrs allemands ont dilué le miel à $5^{\circ} \mathrm{p}$. Ioo dans le sérum physiologique dans leurs expériences et c'est cette dilution qui était chauffée et non le miel pur pour rechercher l'influence d'une élévation de température sur l'inhibine. Or ils admettent eux-mêmes qu'une destruction de l'inhibine à température ordinaire est très rapide si le miel est dilué dans le sérum physiologique. Nous reviendrons d'ailleurs sur cette questions lors de l'exposé de nos travaux personnels. Les auteurs précités ont également montré que la présence d'inhibine n'est pas constante dans les miels et qu'elle peut même manquer totalement. Des épreuves témoins ont été effectuées avec du "miel artificiel » (mélange de glucose et de lévulose rappelant la composition d'un miel). Ce miel artificiel (cru ou chauffé même en concentration de $26 \mathrm{p}$. Ioo dans le milieu, n'est jamais inhibiteur pour le développement des bactéries.

Lorsqu'en I956, 110us avons repris quelques essais sur la valeur antibiotique du miel, nous avons été frappé très vivement par le fait que des extraits acétoniques de miel, mélangés à un milieu de culture et autoclavés $\mathrm{I} / 4$ d'heure à $120^{\circ}$, empêchaient le díveloppement des bactéries ensemencées sur ce milieu.

Cependant, tous les travaux antérieurs mentionnaient une thermolabilité de l'inhibine. Nous avons travaillé pourtant an moins pour une part, avec les mêmes souches que les auteurs précédents, il convenait donc d'étudier cette question plus en détail.

\section{B. - TRAVAUX PERSONNELS.}

\section{Io Méthodes employées.}

Dans les premières séries d'expériences, nous nous servions de l'extrait acétonique de miel. I e miel était trituré, à froid dans un mortier, avec son volume d'acétone. I'opération était renouvelée par trois fois. La totalité de l'extrait obtenu était ensuite évaporé et repris par l'eau. Cet extrait en phase aqueuse était ajouté à un milieu de culture conlé en tubes par quantité de $\mathrm{IoCm}^{3}$. Ce milieu était composé de :

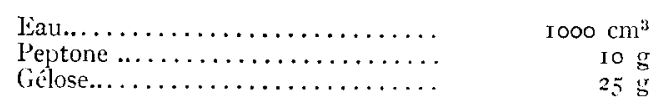


L'ensemble était ensuite autoclavé (à $120^{\circ}$ pendant $\mathrm{I} / 4$ d'heure) ou non avant l'ensemencement et l'on notait les résultats après i2 $\mathrm{H}$ à l'étuve à $37^{\circ} \mathrm{C}$. L,es différences obtenues étaient négligeables entre les extraits autoclavés et ceux qui ne l'étaient pas. Ces résultats portaient sur une trentaine de souches bactériennes répertoriées au tableau III.

C'est alors que, d'autre part, nous avons trouvé que les extraits alcooliques de miel sont beatcoup plus actifs que les extraits acétoniques. La substance antibiotique du miel passe en effet presque en totalité dans 1'alcool, tandis que l'acétone en laisse une certaine partie. Nous utilisons donc depuis plus de I an soit des extraits alcooliques de miel en phase aqueuse, soit du miel pur. Pour mettre en évidence, la différence de la valeur antibiotique des miels crus ou chauffés nous avons employé dans ses grandes lignes la méthode de Dold et Witzenhludsein : on verse stérilement dans des boîtes de Pétri le milieu gélosé (déjà décrit ci-dessus) additionné de miel, ou d'extrait alcoolique de miel (préalablement chauffé ou non). Le mélange est calculé pour obtenir des concentrations correspondant à 25 p. IOO, 20 p. IOO, I 5 p. IOO, IO p. IOO et 5 p. IOO en miel dans le milieu. Les boîtes témoins reçoivent le miel ou l'extrait de miel tandis que les autres reçoivent des extraits ou des miels (suivant l'expérience) soumis à des élévations de température bien déterminées. Après refroidissement on ensemence en surface et l'on porte à l'étuve à $37^{\circ} \mathrm{C}$. pendant I 2 à 24 heures. I'examen après I 2 ou 24 heures permet de noter les résultats et d'évaluer les différences d'action antibiotique entre les miels crus et les miels chauffés. Il est possible de noter l'activité de la substance de o à 5 en se basant sur les données suivantes :

Inhibition avec 5 p. roo de miel dans le milieu note 5 Inhibition avec Io p. Ioo de miel dans le milien note 4 Inhibition avec I5 p. roo de miel dans le milieu note 3 Inhibition avec $20 \mathrm{p}$. Ioo de miel dans le milieu note 2 Inhibition avec $25 \mathrm{p}$. Ioo de miel dans le milieu note I

Pas d'inhibition (avec même $25 \mathrm{p}$. Ioo) de miel dans le milieu note 0 .

Nous pouvons atteindre une précision de 0,25 entre ces notes lorsque l'inhibition n'est pas totale. Précisons encore qu'un milieu témoin additionné d'une quantité de $25 \mathrm{p}$. Ioo d'un mélange de glucose et fructose permet un développement normal des batéries étudiées.

L'un de nous a publié par ailleurs (I) les résultats des essais préliminaires obtenus avec les extraits acétoniques de miel chauffé et par cette méthode, mais nous les laisserons de côté dans cette mise au point, et nous n'exposerons ici que les résultats les plus récents.

(1) Sous presse. 
TABLEAU III

Action antibiotique du miel sur diverses bactéries.

\begin{tabular}{|c|c|c|c|}
\hline \multicolumn{2}{|c|}{ Souches éprouvées } & \multirow{2}{*}{ 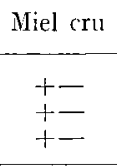 } & \multirow{2}{*}{$\begin{array}{c}\text { Miel chauffé } \\
+- \\
+- \\
+-\end{array}$} \\
\hline $\begin{array}{l}\text { Salmonella pullorum } \\
\text { Salmonella pullorum } \\
\text { Salmonella pullorim }\end{array}$ & 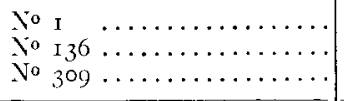 & & \\
\hline $\begin{array}{l}\text { Salmonella gallinarum } \\
\text { Salmonella gallinartmi } \\
\text { Salmonella gallinarum }\end{array}$ & 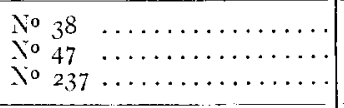 & $\begin{array}{l}+- \\
+= \\
+-\end{array}$ & $\begin{array}{l}+- \\
+二 \\
+-\end{array}$ \\
\hline $\begin{array}{l}\text { Salmonella type Dublin } \\
\text { Salmonella type Dublin } \\
\text { Salmonella type Dublin }\end{array}$ & 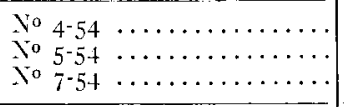 & $\begin{array}{l}+- \\
+- \\
+-\end{array}$ & $\begin{array}{l}++ \\
+- \\
+-\end{array}$ \\
\hline $\begin{array}{l}\text { Escherichia coli } \\
\text { Escherichia coli } \\
\text { Escherichia coli }\end{array}$ & 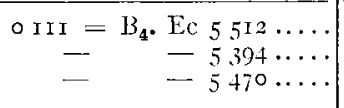 & $\begin{array}{l}- \\
-\end{array}$ & $\bar{z}$ \\
\hline $\begin{array}{l}\text { Escherichia coli } \\
\text { Escherichia coli } \\
\text { Escherichia coli }\end{array}$ & $\begin{array}{cc}55=\mathrm{B}_{5} . & \text { lic } 540 \mathrm{IO} \ldots \\
- & -5423 \ldots \\
- & -5438 \ldots\end{array}$ & $\underline{-}$ & $\begin{array}{l}- \\
-\end{array}$ \\
\hline $\begin{array}{l}\text { Escherichia coli } \\
\text { Escherichia coli } \\
\text { Escherichia coli }\end{array}$ & 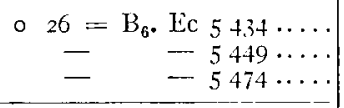 & $\begin{array}{l}- \\
- \\
\end{array}$ & $\begin{array}{l}- \\
- \\
-\end{array}$ \\
\hline $\begin{array}{l}\text { Escherichia coli } \\
\text { Escherichia coli } \\
\text { Escherichia coli }\end{array}$ & 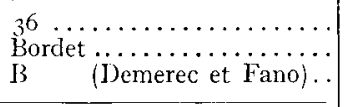 & $\begin{array}{l}++ \\
+- \\
+-\end{array}$ & $\begin{array}{l}+- \\
+- \\
+-\end{array}$ \\
\hline $\begin{array}{l}\text { Proteus vulgaris } \ldots \ldots \ldots \\
\text { Proteus } X \quad 19 \ldots \ldots \ldots \ldots \\
\text { Proteus } V \ldots \ldots \ldots \ldots \ldots \\
\end{array}$ & & $\begin{array}{l}+ \\
+ \\
+ \\
+\end{array}$ & $\begin{array}{l}+ \\
+ \\
+ \\
\end{array}$ \\
\hline $\begin{array}{l}\text { Pseudomonas pyocyanea } \\
\text { Pseudomonas pyocyanea } \\
\text { Pselldomonas pyocyanea }\end{array}$ & 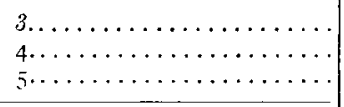 & $\begin{array}{l}+- \\
+= \\
+-\end{array}$ & $\begin{array}{l}+- \\
+= \\
+-\end{array}$ \\
\hline Bacillus subtilis souche $\mathrm{C}$ & ........... & + & + \\
\hline $\begin{array}{l}\text { Bacillus alvei ... } \\
\text { Bacillus larvae... }\end{array}$ & & + & + \\
\hline
\end{tabular}

\section{$2^{\circ}$ Influence du temps et de la température de chauffage sur le facteur antibiotique des miels.}

Nous avons d'abord testé des miels ou des extraits acétoniques de miel chauffés sur une trentaine de souches bactériennes. Lres résultats ont été porté sur le tableau III. Pour toutes les souches considérées un extrait de miel chauffé ou non (autoclavage à $120^{\circ}$ pendant $\mathrm{I}_{5}$ minutes du milieu) était toujours actif.

Nous avons alors étudié l'action des temps et températures de chauffage du miel pur sur la valeur de son facteur antibiotique vis-à-vis de Bacillus subtilis Caron. Il était inutile de travailler sur toutes les souches ce qui compliquerait inutilement le dépouillement des résultats. Les 
souches d'Escherichia coli, par contre ont été soigneusenent évitées. En effet ces bactéries sont sensibles pour certaines doses à l'action d'un facteur de croissance présent dans le miel ce qui rend la lecture des résultats très difficile ou impossible. Ce factetur de croissance présent dans le miel et le miellat fait actuellement l'objet de recherches. Son activité s'exerce principalement sur les souches de Escherichia coli (fait signalé également par SCHUler et Voger en I956).

Les essais ont été répétés avec trois miels différents, mais nous avons volontairement éliminés le:; miels de lavandin dont le comportement au point de vue antibiotique est extrêmement variable. Les trois miels choisis étaient des multifloraux récolté:; à la Station de Montfavet et conservés par nouts-mêmes avec toutes les précautions requises. Il s'agissait :

- d'un miel A récolté en 1958 .

- d'un miel $\mathrm{B}$ récolté au début de l'année I959. l'année).

- d'un miel C récolté en 1959 (mélange de plusieurs récoltes de

Ces miels étaient sensiblement différents mais tous d'origine forale assez variée. Il faut cependant noter que le miel A contenait plus de nectar de lavande et de lavandin que les deux miels $B$ et $C$. La valeur du facteur antibiotique des 3 miels (non chauffés) avant l'expérience était uniformément de 4 .

Nous avons chauffé ces miels pendant $5,15,30$ et 60 minutes aux températures suivantes :

$I^{\circ}$ entre $65^{\circ}$ et $70^{\circ} \mathrm{C}$.

$2^{\circ}$ entre $75^{\circ}$ et $80^{\circ} \mathrm{C}$.

$3^{\circ}$ entre $85^{\circ}$ et $90^{\circ} \mathrm{C}$.

Et pendant I $_{5}$ minutes aux températures suivantes:

$I^{\circ}$ entre $50^{\circ}$ et $55^{\circ} \mathrm{C}$.

$2^{\circ}$ entre $60^{\circ}$ et $65^{\circ} \mathrm{C}$.

Si l'on chauffe à plus de $90^{\circ}$ les résultats deviennent ininterprétables à cause d'un début de caramélisation qui dès cette température transforme alors la nature même du miel.

Les résultats sont consignés sur le tableau IV.

On constate que, chauffés à $50^{\circ}-55^{\circ}$ pendant ${ }_{5}$ minutes le facteur antibiotique du miel n'est pas détruit.

Chauffés à $60^{\circ}-65^{\circ}$ et $65^{\circ}-70^{\circ}$ les miels ont perdu très peu de leur pouvoir antibiotique $\left(\mathrm{I} / 4\right.$ environ à $\left.65^{\circ}-70^{\circ}\right)$.

Chauffés à $75^{\circ}-80^{\circ}$ les mials en perdent un peu plus. Mais sa destruction est encore limitée car après chauffage de $1 / 2$ heure dans ces conditions la note obtenue est encore de 2,5 par exemple. 
TABLEAU IV

Influence de l'élévation de température des miels sur la valeur du facteur antibiotique (éprenve sur B. Subtilis Caron).

\begin{tabular}{|c|c|c|c|c|}
\hline \multirow{2}{*}{$\begin{array}{l}\text { Température } \\
\text { de chauffage }\end{array}$} & \multirow{2}{*}{$\begin{array}{l}\text { Tcmps } \\
\text { de chauffage } \\
\text { en minutes }\end{array}$} & \multicolumn{3}{|c|}{$\begin{array}{l}\text { Note pour le facteur antibiotique } \\
\text { de's différents miels }\end{array}$} \\
\hline & & $A$ & I3 & $\mathrm{C}$ \\
\hline $\begin{array}{l}\text { Miels crus......... } \\
\text { (non chauffés) }\end{array}$ & & + & + & 4 \\
\hline $50^{0}-55^{\circ} \mathrm{C} . \ldots \ldots$ & 15 & + & + & 4 \\
\hline $60^{\circ}-65^{\circ}(\therefore \ldots \ldots$ & I 5 & 3,25 & 3,75 & 3,75 \\
\hline $65^{\circ}-7^{\circ}{ }^{\circ} \mathrm{C} \ldots \ldots$ & $\begin{array}{l}5 \\
15 \\
30 \\
60\end{array}$ & $\begin{array}{l}3 \\
3 \\
3 \\
3\end{array}$ & $\begin{array}{l}3 \\
3 \\
3 \\
2,75\end{array}$ & $\begin{array}{l}3 \\
3 \\
3 \\
2,75\end{array}$ \\
\hline $75^{0.80} \circ 0 \ldots$ & $\begin{array}{r}5 \\
15 \\
30 \\
60\end{array}$ & $\begin{array}{l}3 \\
3 \\
3 \\
2,5\end{array}$ & $\begin{array}{l}2,5 \\
2,5 \\
2,5 \\
2,5\end{array}$ & $\begin{array}{l}2,25 \\
2,25 \\
2,25 \\
2,25\end{array}$ \\
\hline $85^{\circ}-90^{\circ} \mathrm{C} \ldots$ & $\begin{array}{l}5 \\
5 \\
30 \\
60\end{array}$ & $\begin{array}{l}3 \\
3 \\
3 \\
2,75\end{array}$ & $\begin{array}{l}3 \\
2,75 \\
2,75 \\
2,75\end{array}$ & $\begin{array}{l}3 \\
2,75 \\
2,5 \\
2,75\end{array}$ \\
\hline
\end{tabular}

Chauffé à $85^{\circ}-90^{\circ}$ le facteur antibiotique des miels est détruit partiellement mais très irrégulièrement.

I.es graphiques du tableau $\mathrm{V}$ montrent l'influence du temps de chauffage à différentes températures sur différents miels. On remarquera que le miel $\mathrm{A}$ par exemple voit son pouvoir antibiotique diminuer très peu tant que le chauffage ne dépasse pas une demi-heure tandis que les miels $\mathrm{B}$ et $\mathrm{C}$ sont modifiés dès un chauffage de 5 minutes à $75^{\circ} \mathrm{C}$. Il est à remarquer d'autre part que les 3 miels ont le même comportement pour un chauffage à $65^{\circ} \mathrm{C}$., même pendant 5 minutes : ils perdent environs $\mathrm{I} / 4$ de leur pouvoir inhibiteur envers $B$. Subtilis.

Si le chauffage atteint $85^{\circ}$, les graphiques du tableau $\mathrm{V}$ montrent clairement l'irrégularité de l'action de la chaleur sur le facteur antibiotique. Que se passe-t-il alors? Nous ne le savons pas encore, mais l'on peut supposer qu'à partir de cette température élevée, il se produit des modifications biochimiques très importantes.

Dans tous les cas (chauffage pendant 5 minutes; I5 minutes, 30 minutes ou I heure) le miel $\mathrm{C}$ souffre davantage du chauffage tandis que le miel A est le moins modifié.

Lorsque le chauffage des miels dure I heure, les trois courbes du 
$3^{\circ}$ Influence $\mathrm{du} \mathbf{p H} \mathrm{du}$ miel chauffé sur le facteur antibiotique.

Depuis les travaux de DolD, De et Dziao (I938) nous savons que ce n'est pas l'acidité du miel qui joute le rôle de facteur antibiotique et depuis quelques années nous avons nous mêmes constaté que la neutralisation d'un miel n'empêchait nullement celui-ci d'inhiber le développement des bactéries. Cependant nous nous sommes aperçus un jour qu'un échantillon de miel chauffé après avoir été neutralisé à la soude était incapable d'inhiber le développement de Bacillus subtilis Caron.

C'est alors que nous avons testé la valeur antibiotique d'un miel sur Bacillus subtilis Caron et de la manière suivante.

\section{TABLEati VI}

\begin{tabular}{|c|c|}
\hline Miel testé (1) & Valeur antibiotique \\
\hline $\begin{array}{l}\text { Miel naturel non chauffé } \ldots \ldots \\
\text { Miel }+\mathrm{Na} \mathrm{OH,} \mathrm{non} \mathrm{chauffe} \ldots \\
\text { Miel }+\mathrm{Na} \mathrm{OH} \text {, chauflé }{ }^{(2)} \ldots \ldots \\
\text { Miel }+\mathrm{SO}_{4} \mathrm{H}_{2} \text {, chauffe }{ }^{(2)} \ldots \ldots \\
\text { Miel naturel chauffé }\left({ }^{2}\right) \text { témoin } \ldots\end{array}$ & $\begin{array}{l}3,5 \\
3,5 \\
\text { cle } 0 \mathrm{a} \text { I } \\
3 \\
2\end{array}$ \\
\hline
\end{tabular}

Les résultats obtenus montrent que le miel naturel dont on ne modifie pas le $\mathrm{pH}$, ne perd pas la moitié de sa valeur inhibitrice lors d'un chauffage (note 2 au lieu de 3,5 à l'origine).

Si l'on acidifie le miel et que l'on chauffe, la note est plus élevée que si le miel est naturel (note 3 au lieu de 2).

Si le miel est additionné de soude pour le neutraliser, il garde la même valeur antibiotique que le miel naturel (note 3,5) Mais si ce miel neutralisé est ensuite chauffé il perd la presque totalité de son action antibiotique, (note o ou I au lieu de 3,5). Il se produit donc une destruction ou un masquage du facteur antibiotique si un miel chauffé a été neutralisé au préalable.

Nous avons pris un miel de $\mathrm{pH}$ initial 3,8 dont la valeur antibiotique était de 4 (notation de DoLD) à l'origine (miel non chauffé). Nous avons alors modifié (3) le pH de ce miel (en solution dans le sérum physiologique) d'une manière très graduelle et nous avons ensuite éprouvé sa valeur antibiotique avec ou sans chauffage sur Bacillus Subtilis Caron. I Ie chauffage de la solution de miel a été effectué pendant une demi-heure à $80^{\circ} \mathrm{C}$. Après le chauffage, le volume de la solution est réajusté en volume initial grâce à l'addition de sérum physiologique. Les résultats de ces essais sont consignés sur le tableau VII.

(1) Le miel est en solution dans du sćrum physiologique à $50 \mathrm{p}$. 100 .

(2) Chauffage une demi-heure à $80^{\circ} \mathrm{C}$.

(3) Grâce à l'addition de sonde ou d'acide sulfurique. 
On remarque que la variation de $\mathrm{pH}$ n'a ancune influence sur la valeur antibiotique du miel non chauffé.

Le chauffage d'un miel préalablement acidifié n'a pas plus d'influence sur le facteur antibiotique que celui du miel pur $(\mathrm{pH}=3,8)$.

Par contre la neutralisation par la soude permet d'enregistrer après chauffage une chute importante de la valeur antibiotique du miel.

Si l'on arrive à des valeurs dépassant $\mathrm{pH} 7,5$ une deuxième chute de la courbe se produit et la valeur antibiotique (après chauffage du miel) devient quasi nulle.

Si l'on reprend la même expérience en chauffant une demi-heure à $64^{\circ} \mathrm{C}$, , on obtient des résultats très semblables mais dans l'ensemble 1a destruction du facteur antibiotique est moins importante.

D'autres essais nous ont permis également de vérifler que l'on obtient les mêmes courbes (tableau VII) si l'on corrige le pH après chauffage au lieu de le modifier préalablement.

\section{'TABLEAU VII}

Infuence du $p H$ d'un miel sur la valeur du facteur antibiotique

En ordonnée, la valeur antibiotique du miel. En abscisse les $p H$ corrigés (3,8 est le pH initial du miel éprouvé).

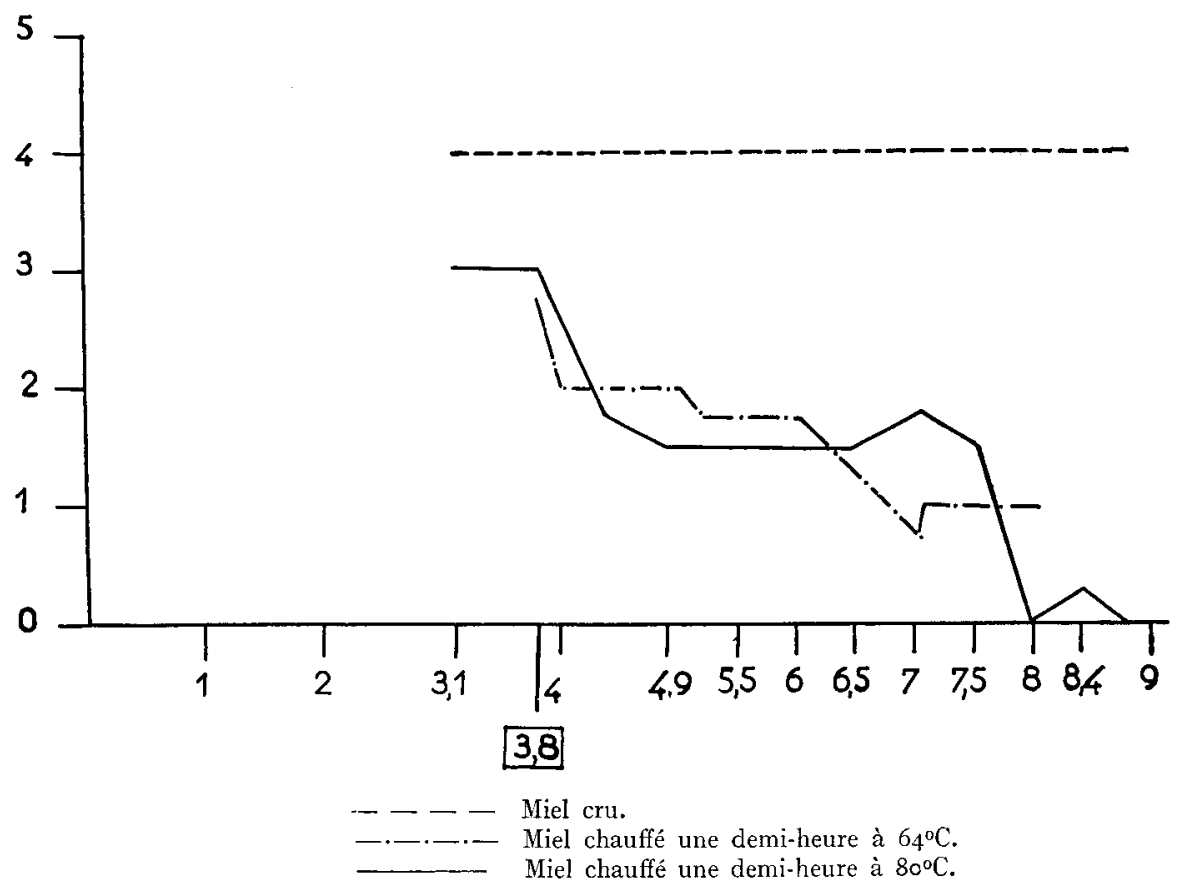


Nous avons essayé également de voir si après une addition importante de soude à une solution de miel il était possible de retrouver l'activité du facteur antibiotique après retour à des $\mathrm{pH}$ assez bas grâce à 1'emploi de l'acide sulfurique. Les résultats obtenus après le chauffage des solutions à $80^{\circ} \mathrm{C}$. pendant une demi-heure sont notés sur le tableau VIII.

TABLEAU VIII

\begin{tabular}{|c|c|c|}
\hline Matériel éprouvé & PH de la solution & $\begin{array}{l}\text { Valeur antibiotique du miel } \\
\text { vis-à-vis de } B \text {. subtilis Caron }\end{array}$ \\
\hline $\begin{array}{l}\text { Solution de miel dans du sérum phy- } \\
\text { siologique }(50 \%) \ldots \ldots \ldots \ldots \ldots\end{array}$ & 3,8 (initial) & 3 \\
\hline Même solution + $\mathrm{Na} \mathrm{OH} \ldots$ & 8,4 & 0,25 \\
\hline Solution à $\mathrm{pH} 8,4+\mathrm{SO}_{4} \mathrm{H}_{2} \ldots \ldots$ & 6,7 & 0,5 \\
\hline Solution à $\mathrm{pH} 8,4+\mathrm{SO}_{4} \mathrm{H}_{2} \ldots \ldots$. & 4,85 & $\mathrm{I}, 75$ \\
\hline Solution à $\mathrm{pH} 8,4+\mathrm{SO}_{4} \mathrm{H}_{2} \ldots \ldots$ & $4, \mathrm{r}$ & 2,75 \\
\hline
\end{tabular}

En conclusion, il est donc possible de retrouver une action antibiotique de cette solution (ajoutée au milieu de culture) si l'on ramène le $\mathrm{pH}$ à ses valeurs initiales.

\section{$4^{\circ}$ Autres facteurs pouvant avoir une influence sur la valeur antibiotique d'un miel chauffé.}

\section{a) INFLUENCE DU SOLVANT POUR LA MISE EN SOLUTION DU MIEL.}

Nous avons déjà vu plus haut que les chercheurs Allemands mettaient leur miel en solution dans le sérum physiologique dans la proportion de 50 p. Ioo avant de l'éprouver. Nous avions pensé que la différence fondamentale entre nos résultats provenait de la méthode employée. Nous avons alors éprouvé la valeur antibiotique du miel pur ajouté au milieu, du miel en solution dans du sérum physiologique $(\% \%$ de $\mathrm{ClNa})$ à $50 \%$ et du miel en solution dans l'eau distillée à $50 \mathrm{p}$. Ioo. Dans tous les cas les résultats sont similaires et la mise en solution n'a pas d'influence sur l'action antibiotique vis-à-vis de Bacillus subtilis Caron. L'action antibiotique d'un miel chauffé est partiellement détruite mais jamais complètement. Par exemple un miel frais de valeur antibiotique 4 passe une fois chauffé une demi heure à $80^{\circ}$ à la valeur de 2,5 quelque soit son solvant, (pur, dans le sérum physiologique ou dans l'eau).

Nous avons trouvé d'autre part qu'une solution de miel dans du sérum physiologique à $5^{\circ} \mathrm{p}$. 100 ne perd pas sa valeur antibiotique à la température du laboratoire et à la lumière. Ceci est contraire au travaux de DoLD comme nous l'avons vu plus haut. Fin effet, une telle solution de valeur antibiotique 4 , conserve sa valeur après roo heures de vieillissement dans les conditions ci-dessus. 
b) INFLUENCE DU CHOIX DU MIEL.

Le choix du miel est d'une grande importance. Par exemple sur certains miels, comme le miel de lavandin, très faiblement antibiotique, l'élévation de température n'a aucune influence sur la valeur du facteur antibiotique.

D'autres miels sont très sensibles à une très brève et faible élévation de température, puis ils ne changent plus de valeur antibiotique pour un temps de chauffage plus long et une température supérieure. Se reporter à ce sujet au tableau $\mathrm{V}$ où le miel $\mathrm{A}$ réagit de cette façon, sa valeur est en effet la même pour des chauffages de $5,1_{5}$, et 30 minutes. I1 faut atteindre un chauffage de 60 minutes à $75^{\circ}$ et plus pour voir baisser la note de 1'antibiotique.

Les miellats ont également un comportement spécial vis à vis d'une élévation de température. Le chauffage provoque une détérioration partielle mais très rapide de l'antibiotique. En effet, nous avons obtenu des destructions importantes de ce facteur après un bref chauffage : par exemple un miellat chauffé 5 minutes à $85^{\circ}$ voit sa valeur passer de 2,75 à $I, 25$.

\section{c) INFLUENCE DE L'ÉLÉVATION DE TEMPÉRATURE}

SUR LE FACTEUR DE CROISSANCE PRÉSENT DANS LFS MIELS,

La présence d'un facteur de croissance dans les miels complique encore 1'étude de la destruction du facteur antibiotique par le chauffage. En effet, nous savons depuis les travaux de SchulER et Voger. (I956) et nos propres constatations (I) qu'il existe dans le miel un facteur de croissance pour les souches d'Escherichia coli qui passe dans la fraction acétonique du mie1. Ce facteur neutralise dans certaines conditions l'action du facteur antibiotique, notamment lors de l'emploi des extraits à des doses faibles dans le milieu de culture. Les deux auteurs précités ont considéré ce facteur de croissance comme thermolabile. Nos essais nous obligent à conclure à sa thermostabilité, cependant nous ne savons pas encore exactement de quelle façon cette substance de croissance réagit au chauffage par rapport au facteur antibiotique et cela nous gêne dans l'étude de ce dernier.

A ce sujet, on pourrait rapprocher ici l'absence d'influence de la température sur le pouvoir modificateur des miels (morphologie, colorabilité et toxicité) par rapport aux bacilles de la diphtérie (DOL,D et KNAPP, I949). Ces auteurs ont montré qu'un chauffage du miel naturel atténue ou détruit son action antibiotique sur Corynebacterium diphtheriae, tandis que "action modificatrice du miel sur ce même bacille est thermostable. Nous n'avons pas réalisé notss mêmes d'expérience sur ce dernier point, mais ils montrent que certaines substances présentes dans les miels ne sont pas toujours détruites par le chauffage.

(1) En cours de publication. 
d) INFLUENCE DU VIEILIISSEMEN'T du MIEL, SUR LE FAC'TEUR ANTIBIOTIQUE.

L'étude de la destruction partielle du facteur antibiotique par le chauffage nous a amené à étudier le vieillisement des miels à différentes températures de conservation. Schade, Marsh et Eckert ont d'ailleurs montré récemment que le facteur antibiotique đu miel était détruit complètement après une conservation de I 5 jours à $+50^{\circ} \mathrm{C}$. Nous avons constaté cependant des différences de valeur antibiotique pour des miels conservés à $+35^{\circ} \mathrm{C}$, à $+20^{\circ} \mathrm{C}$. et à $-6^{\circ} \mathrm{C}$. Les miels conservés $\grave{a}+20^{\circ} \mathrm{C}$ et à $-6^{\circ} \mathrm{C}$ conservent plus longtemps leur valeur antibiotique initiale, mais il n'y a pas de grandes différences entre ces deux derniers après plusieurs mois de vieillissement. La température de $-6^{\circ} \mathrm{C}$. ne bloque pas les transformations du facteur antibiotique du miel au cours du temps.

\section{CONCLUSION}

D'après nos essais récents et les travaux d'autres auteurs nous pouvons dire que de nombreux facteurs peuvent influencer la valeur antibiotique d'un miel soumis à une élévation de température. Les auteurs Allemands qui sont presque tous persuadés de la thermolabilité du facteur antibiotique ont tort à notre avis. D'après nos résultats, le facteur antibiotique du miel (I) est thermostable et lorsqu'il y a une destruction partielle par chauffage celle-ci est en général très faible même à des températures atteignant $80^{\circ}$. Cependant il est non moins certain que la valeur $d u p H d u$ miel joue un grand rôle dans l'altération ou la conservation du facteur antibiotique lors d'une élévation de température. Nous n'avons jamais pu reproduire les résultits obtenus par les auteurs allemands quant à la thermolabilité du facteur antibactérien des miels, même en employant leurs propres méthodes.

Cependant l'interprétation des résultats demande de la prudence ; en effet la variation de la valeur du facteur antibiotique lors du chauffage est influencée par de nombreux facteurs dont il faut tenir compte : choix $d u$ miel, mode d'extraction, "âge "de ce miel et conditions de conservation, présence plus ou moins importante d'un facteur de croissance, présence d'un facteur modificateur sur certaines bactéries. Or si nous avons étudié dans le détail l'influence du $\mathrm{pH}$ et de certains autres facteurs, nous sommes loin d'en avoir épuisé la liste. D'autre part le choix des souches bactériennes et la sensibilité différentielle de certaines bactéries obligent à des mesures très précises.

(1) Vis-à-vis des 30 souches bactériennes du tableau III. 
Dans un travail récent portant sur 600 miels DUISBERG et GEBEI,EIN qui ont étudié l'influence du chauffage sur les miels, n'ont pas fait porter leur enquête sur l'inhibine.

SCHADE, Marsh et ECKERT (I956) montrent que les Allemands ont exagéré l'effet du chauffage sur l'inhibine. F,n effet, un chauffage de 4 heures et demie à $62,8^{\circ} \mathrm{C}$, d'après les auteurs américains, détruit seulement la moitié de l'inhibine d'un miel.

Nous savons également d'après un travail de Maurizio que le miel frais ou bouilli bloque la germination des pollens. Le facteur inhibiteur se confond-t-il avec l'antibiotique du miel? Nous ne pouvons encore répondre à cette question mais l'ensemble des connaissances sur les antibiotiques et les inhibiteurs de germination de pollen ou de graines, permet d'admettre en première hypothèse la parenté des deux facteurs.

Nous pouvons dire d'autre part, que la pasteurisation d'un miel (chauffage bref et refroidissement rapide) agit très peu sur sa valeur antibiotique. Cependant, une étude des différents miels est indispensable pour connaître d'une manière précise le comportement de chacun d'eux vis-à-vis de la pasteurisation. Seule l'expérience pourra nous renseigner sur le temps et la température de pasteurisation convenables pour un miel donné a fin d'éviter la destruction de nombreuses substances et notamment celle du facteur antibiotique. I1 ne sera donc pas possible de pasteuriser les miels sans étude préalable.

Einfin nous conclurons avec Schade, Marsh et EckERT que le principe du dosage de l'inhibine pour reconnaitre un miel chauffé, tel que l'a préconisé 1'Ecole allemande, ne peut pas être retenu comme valable.

\section{RESUME}

Nous avons étudié principalement au cours de ce travail :

\section{L'influence du temps et de la température de chauffage sur le facteur antibiotique de certains miels.}

Les résultats obtenus montrent que le chauffage d'un miel ne détruit pas facilement la totalité de son antibiotique. En désaccord avec les travaux allemands nous avons montré que ce dernier est en grande partie thermostable. Pour des miels chauffés à $80^{\circ} \mathrm{C}$. pendant une demi-heure, 1a valeur antibiotique est encore supérieure à la moitié de celle des mêmes miels frais. Au dessus de $85^{\circ} \mathrm{C}$. l'effet de la chaleur est irrégulier sur le facteur antibactérien; il semble qu'il se produise alors des modifications biochimiques très importantes dans le miel. Le temps de chauffage est très important; après I heure les résultats sont presque semblables sur les différents miels. 


\section{$2^{\circ}$ L'influence $\mathrm{du}$ pH du miel chauffé sur le facteur antibiotique.}

Un miel naturel dont on ne modifie pas le $\mathrm{pH}$ perd environ la moitié de sa valeur inhibitrice lors d'un chauffage à $80^{\circ} \mathrm{C}$. pendant une demiheure. Si l'on acidifie ce miel, l'inhibition se maintient beaucoup mieux, après chauffage. Le miel neutralisé et frais a la même valeur antibiotique que le miel naturel. Mais par contre si le miel est neutralisé et ensuite chauffé il perd la presque totalité de son action antibiotique. Enfin si l'on ramène, après neutralisation, le $\mathrm{pH}$ d'un miel vers sa valeur normale il retrouve ses propriétés inhibitrices vis-à-vis de Bacillus subtilis Caron.

En dehors de ces deux facteurs influençant la valeur antibiotique d'un miel nous avons montré également l'action d'autres facteurs également importants et qui mériteraient une étude plus approfondie. Ces facteurs sont les suivants : Choix du miel, choix du solvant pour la mise en solution des miels, présence d'un facteur de croissance antagoniste du facteur antibactérien sur Escherichia coli, et vieillissement des miels.

\section{REFERENCES BIBLIOGRAPHIQUES}

Dold (H.), Du (D. H.) et Dziao (S. T.). - Nachweis antibakterieller hitzeund lichtempfindlicher Hemmungsstoffe (Inhibine) im Naturhonig (Blütenhonig). Zeit. 7. Hyg., 120, I55-167, I938.

DoLD (H.) et KNAPP (T.). - Uber inhibierende und modifizierende Wirkungen des Honigs auf Diptheriebazillen und seine Brauchbarkeit zur Bekämpfung des Diphtheriebazillenträgertums. Zeit. f. Hyg., 130, 323-334, I949.

Doln $(\mathrm{H}$.) et Wr'Tzenhausen (R.). - Ein Verfahren zur Beurteilung der örtlichen inhibitorischen (keimvermehrungshemmenden) Wirkung von Honigsorten verschiedener Herkunft. Zeit. $f$. Hyg., 141, 333-337, 1955 . Duisberg (H.) und GEBELEIN (H.). - Uber die Kontrolle von Erhitzungsschäden bei Honigen. Zeit. f. Lebensmittel-Untersuchung und-Forschung 10\%, (6), 489-50I, I958.

Franco (M.). - Am. Ig., 51, 394, I94I.

Franco (M.) et Sartori (L.). - Ann. Ig., 50, 2i6, in Presse Med., no 68-69, 716, I940.

Gabbert. - Honig als Antiseptikum. Diss. Med. Würzburg, I936.

Mavrizio (A.). - Substances dans le corps de l'abeille mellifique qui empêchent la germination du pollen. Communication au $X V I I^{\mathrm{e}}$ congrès International d'apiculture. Bologne-Rome, I5-23 septembre I958.

Póthmar (J. H.). - Z. Hyg. Intektionskrankh., 130, 468-484, I95I.

Schade (J.), Marsh (G.) et E.CKERT (J.). - Improved methods of determining diastase and hydroxy-methyl-furfural in honey and their relationship to the bacteriostatic quality of honey. Communication Ioth International Congress of Entomology. Montreal, August 17-25, 1956.

Schuler (R.) et VOGEL (R.). - Wirkstoffe des Bienenhonigs. Arzneimitt. Forsch. 6, 66I-668, I956.

VERGÉ (J.). - L'activité antibactérienne de la propolis, du miel et de la gelée royale. Apiculteur, 95, (6), I3-20 (I0), I95I.

White (G. F.). - The bacteria of the apiary. U. S. départ. of Agriculture Technical series $\mathbf{n}^{\circ} \mathbf{I} 4$, Igo6. 